\title{
A new characterization of scientific theories
}

\author{
Jody Azzouni
}

Received: 1 November 2009 / Accepted: 27 April 2011 / Published online: 16 May 2014

C) The Author(s) 2014. This article is published with open access at Springerlink.com

\begin{abstract}
First, I discuss the older "theory-centered" and the more recent semantic conception of scientific theories. I argue that these two perspectives are nothing more than terminological variants of one another. I then offer a new theory-centered view of scientific theories. I argue that this new view captures the insights had by each of these earlier views, that it's closer to how scientists think about their own theories, and that it better accommodates the phenomenon of inconsistent scientific theories.
\end{abstract}

Keywords Semantic conception of scientific theories - Inconsistent theories Formal languages $\cdot$ Scientific theories as models $\cdot$ Bridge principles

\section{Introduction}

I first present two "received views" of scientific theories. Both views can be characterized in terms of Tarski's (1983) semantics for logical formalisms. ${ }^{1}$ For concreteness, consider a first-order language Lan with an unspecified number of nonlogical constants and predicates. Theories are collections of sentences of Lan. A domain $\mathrm{O}$ is a set of objects. In standard presentations of model theory, a model of a first-order language Lan is a pair $(\mathrm{O}, \mathrm{M})$, where $\mathrm{O}$ is a domain, and $\mathrm{M}$ is a mapping of the nonlogical constants of Lan to elements of $\mathrm{O}$ and the nonlogical $n$-place predicates of Lan to $n$-relations of the elements of $\mathrm{O}$.

Given a Tarskian recursive definition of truth-in-a-model, we can characterize scientific theories in two possible ways: as formal theories (sets of formalized sentences)

\footnotetext{
1 The semantic conception self-consciously arises from Tarski's work. Theory-centered views officially predate Tarskian semantics but (as I show) are easily accommodated in its framework.
}

J. Azzouni $(\bowtie)$

Tufts University, Boston, Massachusetts, USA

e-mail: jody.azzouni@tufts.edu 
or as collections of models (set theoretic, or otherwise). Proponents of (earlier) theorycentered approaches often describe a scientific theory as a set of laws $L$ from which the remaining sentences of that scientific theory deductively follow. Any such set of laws has a family of models that it's Tarski-true in. One-or several—of these are the intended models of the scientific theory. Sometimes the intended models are taken to be characterized by correspondence rules that map the nonlogical vocabulary to items in the world.

A broader more flexible way of characterizing a range of intended models in theory-centered approaches is by parameters. These are specified nonlogical constants and relations that are empirically fixed (e.g., numerically: the fundamental constants of physics; or ontologically: as referring to this or that item or relation or to any such in a specified class). Further flexibility is achieved by treating a scientific theory as containing law-schemata instead of lawful sentences (with or without parameters). ${ }^{2}$

It might be thought that even more flexibility is introduced if the theories in question are allowed additional resources by deserting the first-order framework altogetherby allowing theories to be couched in higher-order logics, for example. It might even be argued that scientific theories and mathematical theories themselves are already higher-order formalisms that clearly involve quantification into predicate positionsand that this is the way to understand informal-rigorous mathematical characterizations of well-ordering, for example. ${ }^{3}$

In response, I should first point out that the increase in expressive power the introduction of higher-order logics apparently brings is spurious - that it does not go beyond the actual representational capacities of theories in first-order languages. See footnote 12 below for indications for why this is true and for references. Second, as I will indicate towards the end of Sect. 2, it's a mistake to characterize scientific and mathematical theories - as they occur in ordinary mathematical and scientific practice-as formalisms, either first-order, higher-order, or whatever. Rather, they are written in natural languages supplemented with additional technical vocabulary. It is, therefore, a theoretical reconstrual of scientific theories (a more or less justified theoretical reconstrual) to characterize them as formalisms. This point will be important towards the end of this paper. Regardless of these two points, nothing in theory-centered formalization approaches to scientific theories requires first-order logic; and so readers should understand the language of theories according to theory-centered approaches to be open-ended with respect to the logic they presuppose. Thinking of them as so openended will prove valuable when theory-centered approaches are compared to semantic approaches in Sect. 2.

On some versions of theory-centered approaches, $L$ is to characterize the laws of everything (other than abstracta). On this attractively simple "unity of science" picture, the domain of a scientific theory is everything — or everything fundamentaland its nonlogical predicates correspond to the law-relevant relations of those objects. Such a theory is false if it's not Tarski-true in the world: if the (physical) items of

\footnotetext{
2 See (Kuhn, 1970, p. 188) and (Azzouni, 2004, pp. 18-19).

3 My thanks to the anonymous referee for pressing me on this point.
} 
the world and their law-relevant relations isn't one of its models. Inducing successor scientific theories involves changing sentences from previous scientific theories with consequent changes in the family of models of such theories.

Many opponents of theory-centered approaches don't recognize the enormous flexibility of such approaches, that (for example) a global realist-representational characterization of scientific theories isn't required by the mere assumption that scientific theories are linguistic entities. For example, the domains of scientific theories-on theory-centered views - needn't contain everything that exists: domains can be small. Although theory-centered approaches (for certain logical positivists) were understood as programmatically characterizing a unified science with one (physical) domain, theory-centered approaches are compatible with a very different picture: The sciences are highly disunified and different sets of laws hold in different domains. Indeed, the domains in question can either be physically disjoint from one another, or they can instead be characterizations of a given phenomenon in terms of entities at different levels of "grain."

Furthermore, although I initially described theory-centered approaches as attempting true descriptions of the items in the world-and concomitantly, as attempting "realist" representations of what there is - that's not required either. Theory-centered approaches are compatible with anti-realism: the view that scientific theories aren't true but have other virtues, "approximate truth" or "predictive value," etc.

Too many of the polemics directed against theory-centered views have focused on subsidiary positions held by some of their adherents, or by philosophers labeled vaguely as "logical positivists," but that aren't essential to theory-centered approaches. Another example is this: Proponents of theory-centered approaches saw the applications of a scientific theory as arising by the logical deduction of consequences from sets of laws. Where the application-area is characterized in a vocabulary different from that of the laws themselves, they invoked bridge principles as providing the deductive medium linking statements in the two vocabularies. Relatedly, they regarded a scientific theory as deductively linked to the more specific theories that arise from it. This deductivist perspective, however, isn't required by the assumption that scientific theories are linguistic entities. Other ways - compatible with theory-centered views-are available to explain how scientific theories are applied, and how scientific theories relate to their specifications. ${ }^{5}$

Let's turn now to the other received view. ${ }^{6}$ This second family of views-the semantic conception-instead takes scientific theories to be collections of set-theoretical models or (more vaguely) nonlinguistic structures of some sort. ${ }^{7}$ State spaces (Beth

\footnotetext{
${ }^{4}$ For example, as persons or as cells or as collections of molecules.

5 I omit further discussion of deductivism. See Azzouni (2000, especially Part I).

6 There are many proponents, and so many versions, of semantic approaches. See Suppe (1989), Prologue, for history, and Suppe (1989, 35, footnote 2) for references. Some proponents: Van Fraassen (1980), Suppe (1989), Sneed (1971), Stegmüller (1976), Giere (2000); but there are many others. See Frisch (2005, 195, footnote 2) for further references. The approach originates in Beth (1948).

7 That scientific theories are nonlinguistic entities is meant literally - at least by many although not all proponents of the semantic conception. Giere (Giere (1988, p. 85): "we understand a theory as comprising two elements: (1) a population of models, and (2) various hypotheses linking those models with systems in the real
} 
and van Fraassen), or relational systems (Suppe) are some examples. Further, these structures needn't be purely mathematical but can either contain actual items from the world (e.g., physical entities) or (more vaguely) "phenomena." Where such a collection can be characterized as the models of a formal theory, of course, there doesn't seem to be any difference between taking a scientific theory to be that formal theory and taking the scientific theory to instead be the models of that theory. ${ }^{8}$ But, some have argued, such a collection of structures can't be so characterized because the structures in question (although similar in vocabulary) can fail to be "axiomatizable" — that is, there is no (recursive) characterization of the sentences true in all and only those structures. I discuss this claim further in Sect. 2.

As noted, the semantic conception is credited with a flexibility and an accuracy towards ordinary scientific practice that's denied to theory-centered approaches. ${ }^{9}$ In particular, proponents of the semantic conception claim (i) that the semantic approach better fits the view (of scientists) that the scientific theories in any field are "tools" to solve problems rather than true descriptions of the world and its laws. They also say (ii) that scientists treat theories as "models" of a phenomenon, and that this practice is better characterized by the "models" of the semantic conception than by anything linguistic. Finally (iii), they sometimes claim that the individuation conditions for theories, when construed linguistically and when construed model-theoretically, are different, and that the individuation conditions for theories construed model-theoretically fit the individuation conditions that scientists use better. ${ }^{10}$ I've already indicated reasons to doubt (i) and (ii), but I discuss all three claims further in the following sections.

\section{What do intended models show?}

Van Fraassen (1985, pp. 301-302) and Van Fraassen (1989, pp. 210-213) defends the semantic conception of theories against the claim that the distinction between semantic and formalization approaches to such theories is "a distinction without a difference." 11 He argues that scientific theories can be (and are) presented as sets of models, but that this practice evades formalization because of the nonstandard models of pertinent first-order theories. For example, if each of a set of models of some

\footnotetext{
Footnote 7 continued

world." Suppe (1989, p. 3): "According to the Semantic Conception of Theories, scientific theories are not linguistic entities, but rather are set-theoretic entities." Stegmüller (Stegmüller (1978, p. 39): "Physical theories are introduced as sets of ordered pairs, each pair consisting of a sequence of mathematical structures and a class of domains of applications." Van Fraassen (1989, p. 222): "if the theory as such, is to be identified with anything at all —if theories are to be reified - then a theory should be identified with its class of models." Suppes (1967), however, doesn't identify a scientific theory directly with a collection of models, and da Costa and Da Costa and French (2003, pp. 33-34) offer a complex extrinsic/intrinsic picture with interplay between viewing a scientific theory as a collection of models and viewing it as a linguistic characterization of such a collection.

8 As noted by Friedman (1982).

9 Stegmüller (1976, Chap. 1) and Suppe (1989, Chap. 2), Nickles (2002) and Da Costa and French (2003).

10 Suppe (1989, p. 4) and Da Costa and French (2003, p. 24).

11 This charge occurs in Friedman (1982) and in Worrell (1984). I'm revisiting it, and sustaining it, against Van Fraassen's (1985), and Van Fraassen's (1989) subsequent defense.
} 
phenomenon includes the real number continuum, any first-order formalization meant to capture that set of models will also include nonstandard countable models. Against the natural suggestion that a first-order formalization approach can be restricted to the "intended" or "standard" models, Van Fraassen (1989, p. 212) denies that such descriptive restrictions are available to "syntactic" approaches "except by fiat."

What this defense relies on-as Van Fraassen (1989, pp. 210-213), makes clearis the substantial claim that there are linguistic resources in natural languages for fixing reference to intended models beyond what's available in formal languages. This issue arises rather directly in philosophy of mathematics. Several authors-e.g., Myhill (1951) and Pearce and Rantala (1982), among others — suggest that the apparent ability of mathematicians to refer (only) to intended models is due to resources in natural languages, but not in formal ones.

Van Fraassen (1989, p. 212) also claims that the resources in natural languages can't be captured by formalisms because "natural language consists in the resources we have for playing many different possible language games," resources that formal languages presumably don't have. This, however-even if right-merely raises an issue. The intuitive impression (in informal mathematical practice) that one refers to intended models makes acute the question of how one does this. Indeed, as I indicate in the next two paragraphs, such reference in natural languages involves acts of "fiat" that are identical in nature to what's available to formalist approaches for reference.

For consider the case van Fraassen mentions, the "presentation" of intended collections of uncountable models without the inclusion of countable ones. The data is that ordinary practice "enables" this_routinely_by descriptions of the intended models in the vernacular coupled with specialized vocabulary. But anything present in ordinary language or available to its users that enables them to fix on intended models either fails at this or can be similarly employed by users of formalized languages. If, for example, it's the intentions or other cognitive states of the mathematician/scientist that enables such reference, these can similarly be enjoyed by users of formalizations. If there are specialized idioms in the vernacular with referential powers beyond first-order idioms, then we can also invent formalizations using similar idioms. In particular, if higher-order quantifiers occur in natural language that enable the formulation of theories that don't have the unintended models that first-order theories have, then they can obviously be incorporated into formulations of scientific theories in a way that's compatible with theory-centered approaches.

Finally (and this exhausts the options), if it's the meanings of mathematical terms that force reference to intended models and not to other models, these too can be transplanted to a formal setting via "meaning postulates." In short, one shouldn't assume, as van Fraassen does, that however it is that mathematicians and other scientists "present" exactly the mathematical structures they want to present (and not other unintended ones), it's by (unexplicated) resources that elude formalization. ${ }^{12}$

\footnotetext{
12 Van Fraassen's word "fiat" strikes exactly the right note with respect both to formalisms and to ordinary language. I argue in my 1994, Part I, § 3, that the incorporation of any linguistic resources in a formalism to enable the exclusion of unintended models relies on mere fiat to manage that magic. In other words, resources that go beyond the first-order case in their referential strength involve forms of buried fiat to do so. So the right conclusion isn't that ordinary language has resources that formalisms lack; it's that various forms of buried fiat are doing the same job in ordinary language.
} 
Some proponents of the semantic conception think the general absence of formalizations from ordinary scientific practice supports their position. Suppe (1977, p. 221) claims, for example, that because formalizations are rare in science, scientific theories can't be collections of propositions or statements, but must therefore be nonlinguistic. All that really follows, of course, is the truism that the propositions or statements in question are denizens of ordinary language (supplemented with specialized vocabulary). It's equally true that ordinary mathematics occurs in natural languages supplemented with specialized vocabulary, and not in a formalized setting. (See my 2009 (a), and the other articles cited there.) It would be bizarre, however, to draw the conclusion in the mathematical case that Suppe draws in the scientific case: that, therefore, mathematical theories are extra-linguistic entities.

Given the foregoing, the immediate corollary is that the semantic conception can't involve anything distinctive that's unavailable to theory-centered approaches. This is because any presentation of a family of models must involve (theoretical) descriptions, and that means that scientific theories can be always be identified with those (theoretical) descriptions instead of with the family of models those descriptions characterize. It's important to stress, however, the point just made that scientific theories aren't de facto formalisms but instead are linguistic entities of ordinary language supplemented with a specialized vocabulary. This point deviates from both perspectives on scientific theories that I initially described in Sect. 1. It's also important to stress that this is true not only of scientific theories but also of mathematical theories (as such theories occur in ordinary mathematical practice). Mathematical theories are written in the vernacular augmented with additional technical vocabulary, and therefore it is a reconstrual of them to characterize them either as first-order theories or as higher-order theories.

Although these facts raise interesting and difficult questions both about the relationship of scientific theories and mathematical theories to ordinary language and difficult questions about their relationship to the formalisms that philosophers use to describe them, nothing in the analysis of these questions yields anything that's particularly supportive of the semantic conception of scientific theories.

In Sect. 4 I will use the fact that scientific theories occur in the vernacular and not as formalized theories to illuminate aspects of scientific practice with respect to inconsistent theories. Meanwhile, however, I'll return to defending the flexibility of theory-centered views against their semantic conception competitors.

\section{A new characterization of scientific theories}

In order to address issue (iii), the claim that how scientific theories are individuated according to theory-centered views doesn't fit with how scientists individuate their own theories, I first need to sketch a theory-centered view different from standard ones. $^{13}$

\footnotetext{
13 Previous discussions of my version of a theory-centered view of scientific theories occur in my 2004, Chap. 2, and in my 2009 (b).
} 
For purely illustrative purposes, let's again treat scientific theories as collections of first-order sentences that scientific practitioners sometimes take to possess terms with fixed interpretations, and sometimes to possess terms open to varying interpretation (relative to a context of application). ${ }^{14}$ To begin with, the sentences of any scientific theory contain constants and predicate expressions. Let $c_{1}, \ldots, c_{m}$ be the constants, and $P_{1}, \ldots, P_{q}$ the various $n$-place predicates, $1 \leq m, q$, of a scientific theory $L$. An interpretation $(\mathrm{O}, \mathrm{M})$ of $L$ is a model of the language of $L$, as in Sect. 1, except thatgenerally - not all of the objects in the domain $\mathrm{O}$ are real. Thus, some-but generally not all - of its constants $c_{1}, \ldots, c_{i}, 0 \leq i \leq n$, are mapped by $\mathrm{M}$ to items in the world. The rest of the constants are empty. ${ }^{15}$ Similar remarks apply to the $n$-place predicates. I presuppose sentences with empty constants to nevertheless have truth values. The notion of "truth" in play, therefore, is deflationist, and not a "correspondence" one. More accurately, the presupposed notion of "truth" is compatible with correspondence, but only when all the constants in a sentence are nonempty. I refer the interested reader to other work for further details and arguments. ${ }^{16}$

In any case, most of the theories scientists use are recognizably false of the domains they are applied to. That is, not all of the implications of such theories are true. Consider $T H$, the set of implications of some scientific theory $L$. What makes $L$ valuable, with respect to an interpretation, are (some of) its true implications. These true implications are singled out by description, so that a scientist, when utilizing a scientific theory, commits herself only to the truth of the useful implications of $L$ (and not to the rest of it).

A toy example. Consider Ptolemaic astronomy $(P t)$. Imagine that the constants of $P t$ are interpreted as referring to the planets, the luminaries, various geometrical objects, etc. $P t$ also has predicates interpreted as characterizing positions of moving bodies, classes of mathematical functions, etc. The constants that refer to the various planets are nonempty; most of the other constants are empty. In addition, $P t$ is false: it doesn't describe the real locations of bodies. However, the apparent-location consequences of $P t$ (as projected onto a celestial sphere from the vantage point of the Earth) are true of those bodies, and it's those consequences that make $P t$ valuable. ${ }^{17}$

\footnotetext{
14 Treating scientific theories as linguistic entities doesn't exclude their being abstracta (types), e.g., ordered types that contain the objects and relations they are about (i.e., "Russellian" propositions). It also doesn't exclude an individuation of "sentences," so that different syntactic types express the same "statement" as in natural languages. My discussion is neutral about this.

15 In my view, no interpretation of a scientific theory has only nonempty constants. This is because most-if not all-scientific theories have at least some constants that refer to mathematical entities. All such, on my view, are empty: there is nothing real they refer to. Alternatively put, "refer," when used with respect to such constants, isn't a genuinely relational word. "Not everything in the domain $\mathrm{O}$ is real" isn't meant to express a Meinongian position where the domain $\mathrm{O}$ is to contain both real and unreal entities. Rather, talk of "domains" is (as it always is) a metaphor standing for a particular kind of (Tarskian) semantics with truth-conditional relations posited between object-language terms and metalanguage terms. Just as certain object-language terms are empty, so are the corresponding metalanguage terms. This is an ontologicallyneutral use of Tarskian semantics. See my 2004, 53-55, or my 2010, Chap. 5, for further discussion.

16 Azzouni (2006, 2010), forthcoming (a).

17 Many philosophers appeal to "approximate truth.” But it's not needed. Here's a sketch of the argument: Any time "approximately true" characterizes a successful scientific theory, that theory can instead be characterized as a false theory with certain true implications. Specifically, that a certain numerical implication
} 
The interpretation of $P t$ involves nonempty constants. We can say, therefore, that certain implications of $P t$ - the apparent-location implications - are true of the entities referred to by those nonempty constants. Many scientific theories are similarly true of entities we think are real. Many others, however, aren't. A scientific theory can often be successfully applied to phenomena where, given how it's interpreted, all of the constants in the theory are empty. (There is nothing real in the application-area corresponding to the entities in the domain of that theory.) For example, a successfully applied scientific theory may refer, apart from mathematical entities, only to magnetic monopoles, or to other virtual particles.

There is one last wrinkle. I've utilized the word "interpretation" to describe how the references of the constants of a scientific theory may be fixed as referring to certain items in the world, and correspondingly, how its predicates can be fixed in the relations they correspond to. What's important to note now is that-and proponents of the semantic conception have rightly stressed this - a scientific theory is often characterized apart from any particular interpretation. This ubiquitous practice is evident right from the beginning of one's study of physics. A theory, characterized by a set of Newtonian $n$-body equations, say, is developed in a purely mathematical manner. It's then applied in numerous cases. Sometimes, in an application, the constants of the theory are interpreted as not being empty: e.g., as the Sun and Jupiter. Sometimes, in an application, all the constants are empty: they refer only to various virtual particles and purely mathematical entities. Regardless, in any case where a scientific theory is applied successfully, it has useful true implications. What's crucial to note in this kind of case, however, is that the scientific theory is not individuated in terms of its applications (or by how the references of its constants and predicates are fixed in these applications). Rather, it's understood that the "same" theory is applied in different cases. It's here that the scientist's use of "model" to characterize the role of scientific theories becomes natural. The application of the theory allows scientists to "model" the phenomena in terms of the theory.

It's important, however, not to reify "models." Proponents of the semantic conception do exactly that: treat models_-so described-as mathematical entities, and so they assimilate this use of the word "model" to a quite different case where actual physical models of something are made (e.g., physical models of proposed buildings). The scientific use of the word "model" is natural because of the various simplifications the applications of many scientific theories impose on an otherwise complex phenomenon, e.g., the interactions of physical entities being treated as the interactions of rigid

\footnotetext{
Footnote 17 continued

of a theory is "approximately true" can be characterized as the truth that a certain measured value is within $\pm n$, for specified $n$. For example, where Newton's laws are currently still applied, it's not because those laws are "approximately true," but because those (false) laws have implications that are true tout court. The technical fact crucial to this argument is that adjectival constructions modifying "true" (e.g., "approximately true that so and so is such and such") can be pushed through the truth predicate to modify instead the contents following that predicate (e.g., "true that so and so is approximately such and such").

It's often overlooked, I think, that Newton's laws not only imply precise numerical results in such and such situations; but those very numerical results themselves imply (various) approximate numerical results that are also implied by general relativity in such and such same situations. In this way, in general, some of the implications of a succeeded theory can nevertheless be true (and not approximately true!) from the vantage point of a successor theory because they are implied by that latter theory.
} 
bodies under simple force assumptions. My interpretation is that, in all these cases, one or another scientific theory is applied to a phenomenon even though (and this is crucial), the theory is actually false of the phenomenon. The reason for doing so is that the theory in question yields a number of useful true implications when interpreted as applying to that phenomenon. Imagining the "entities" of a theory independently of its applications, however, invites thinking of it as a "model"- - kind of structural object that has an independent existence, and the entities of which can be taken to "represent" the machinations of the phenomena in a simplified way. But this way of approaching theories has no content beyond a theory being interpreted to enable an application that is successful precisely because it yields a number of true implications.

Let's now turn to issue (iii), that theory-centered approaches to scientific theories supposedly fail to individuate those theories as scientists do. I'll leave aside the evident point (in my favor) that the individuation of scientific theories is, in any case, a murky one. There is no bright line that indicates why certain changes in a theory (or in its application) incline practitioners to describe the result as a new theory, rather than as a modified version of the same theory. Surely sociological factors play the major role here.

Proponents of the semantic conception, however, regard theory-centered approaches as badly off with respect to individuation, even apart from the above point. This is because they take theory-centered approaches to require both that there be "correspondence rules" that indicate what scientific terms refer to, and that the individuation conditions of scientific theories be sensitive to those rules. Suppe (1989, p. 4) and Da Costa and French (2003, p. 24) argue that since such correspondence rules must be characterized in terms of "experimental procedures" and "measurement techniques," both of which change constantly, theory-centered views are saddled with the absurd result that new scientific theories emerge from older ones just by the refinement of measurement techniques.

The "theory-centered approaches" so attacked are "straw men." Talk of "correspondence rules," in any case, is naïve. The correct idea, of course, is that the applicationareas of scientific theories need to be indicated in some way. Those (early) philosophers who labored under the (false) impression of there being a sharp theory/observation distinction thought that correspondence rules were needed to anchor a theory entirely couched in non-observational vocabulary; otherwise nothing in the theory could indicate how or where it's to be applied. But there is no genuine issue here. Any scientific theory-like any collection of sentences of ordinary language-has grammaticallyreferring expressions. And there are two possibilities to consider, when the referring expressions refer to real objects, and when they don't. First case. Some of the referring expressions of a theory are taken to be real objects. Then nothing like "correspondence rules" arises, no more than with the words of ordinary life. Rather, one takes one's instruments, observations, and so on, to defeasibly describe truths related in various ways to those objects, and those truths are then utilized directly with the theory itself. This is the case regardless of whether the theory is taken to be true or false of the objects that it's applied to. ${ }^{18}$

18 Imagine that one has a certain theory about cows. One tries to confirm that theory by observing cows, or by other (instrumental) ways of learning about cows. One may develop auxiliary theories about how 
Second case. A scientific theory is applied to a phenomenon even though none of the entities referred to by terms in that theory occur in that phenomenon (e.g., continua in the case of materials). Here too, certain instrumental interventions and other ways of gathering data will (defeasibly) be treated in terms of the theory-as indicating that such and such is the case with the entities the theory posits. Perhaps the phrase "correspondence rule" can be made to better apply here-but it gives the false impression that the application of a scientific theory, even in this case, involves some kind of rule. It's hard to see what the rule could be. Rather, one imposes a theory on the phenomenon in a certain way by provisionally interpreting various instrumental events as indicating certain truths couched in the language of the theory. One hopes that doing so will result in implications that predict phenomena. In any case, it's unreasonable to treat such interpretations as constitutive of the identity conditions of the scientific theory (when construed linguistically). In particular, a theory-centered approach needn't be hostile to the idea that some scientific theories are to be individuated in a way that treats them as having a quite open-ended class of applications.

It's true that many early proponents of theory-centered approaches treated scientific theories as fixed in their references, and that those philosophers thought that scientific theories were designed only to truly describe something or other. There can be no denying that some scientific theories do have that job (or at least, have that job when so interpreted by scientists): adherents of the semantic conception overlook this fact. But those adherents are right to stress that many scientific theories have another job: not to describe a phenomenon truly, but to be a tool that extracts truths from what's otherwise an intractable area of study. To this end, the semantic conception pictures scientific theories as collections of (simplified) models of a phenomenon-a toolkit. This picture, however, overlooks the (linguistic) material out of which the tools in this kit are actually constructed: language. The tools, that is, are false theories with true implications. And it's very important to realize that scientists think of these theories in just this way. $^{19}$

It's an interesting question whether (nowadays) any scientific theory is regarded as (defeasibly) true tout court. Various branches of Newtonian physics were certainly once so regarded; but whether this is true of any contemporary physical theory may well be doubted. ${ }^{20}$ In any case, even if we (collectively) regarded all our applicable

\footnotetext{
Footnote 18 continued

one observes cows (theories of vision), or one may not. The situation is no different even in physics. The application of the instruments utilized in physics almost always involves one scientific theory or anotherbut our ability to apply them often goes beyond any theory in various ways. See Azzouni (2000, Part I.) In neither case, in ordinary life or in the sciences, are "correspondence rules" utilized.

19 Some scientific theories are treated both ways (although in different contexts). That is, the same syntactic forms can be taken to be true tout court when applied to one kind of phenomenon, and can be treated as tools that are false when applied to other phenomena.

20 Perhaps certain appropriately restricted physical theories are taken to be true tout court. This doesn't seem to be the case, however, with our two contemporary grand theories: general relativity and quantum mechanics. The current search (among certain physicists) for a unified successor theory that can be applied everywhere indicates this.
} 
scientific theories as false tout court that would still leave us with plenty of truths-it would just be that access to such truths would have to come packaged in scientific theories that were (as a whole) false. ${ }^{21}$

One last observation. Many philosophers have described cases where incompatible assumptions are used to derive different aspects of the same phenomenon. Nickles (2002, p. 3) notes of Ptolemaic astronomy that "the constructions used to predict the size of the moon at a given time were incompatible with those used to predict its location or its speed." Cartwright (1983) discusses similar examples from contemporary physics. These cases are easily handled in accordance with ordinary scientific beliefs about the matter: that the theories (or specific "laws") in question are false, but have certain true implications. The true implications must be mutually compatible; but this isn't true of the false theories used to derive them.

\section{Contradictory scientific theories}

The foregoing discussion indicates that one reason some false scientific theories are indispensable is because they can be exploited to extract needed true implications (about a phenomena) that can't be derived from a true theory, either because no true theory with such implications is available, or because any true theory that would imply these results isn't computationally tractable, or requires empirical parameters that are impossible to determine, etc. One derives implications from false scientific theories according to any (truth-preserving) inference method that works and can be applied to it. All that's needed is a characterization that circumscribes the true implications, e.g., "all the implications of $L$ about the strength of the magnetic field are true," or "all the implications of $L$ about the second-order effects of such and such are true," etc.

Scientific practice, however, reveals not only that indispensable theories known to be false are ubiquitous, but that even theories known to be inconsistent are common. ${ }^{22}$ How and why are these useful for scientific practitioners? The why is easy: there is often no consistent theory-not even a false one-available from which particular valuable implications can be drawn. The how is the important question, especially since in classical-logic settings an inconsistent set of sentences implies everything. Second, there is an important puzzle about what practitioners take themselves to be doing when they work with theories or collections of theories that they know to be inconsistent. I've argued elsewhere that natural languages are trivially inconsistent ${ }^{23}$;

\footnotetext{
${ }^{21}$ Further: even if all scientific theories were false, it wouldn't be that low-level "phenomenological" generalizations were the only truths we had. High-level generalizations of narrow scope (about highly theoretical entities) would also be true. The primary difference between my view and Cartwright (1983) is that she takes a theory as false if it isn't empirically confirmed, whereas I think the indispensability of a scientific statement (or theory) can suffice for our having to take it to be true-confirmation needn't come into it. See Azzouni (2009(b)).

22 A taste of the literature: Brown (1990, 1992); Frisch (2005); Meheus (2002a); Norton (2002); Smith (1988). See the opening paragraph of Da Costa and French (2002) for a list of prominent scientific theories that are taken to be contradictory.

${ }^{23}$ In particular, I argue that natural languages obey a logic that is classical, and so the contradictions of natural languages make them trivially inconsistent: Every statement in such languages is both true and false. See Azzouni $(2003,2007)$.
} 
an important element of my description of how ordinary speakers navigate trivially inconsistent languages, however, is that they are unaware natural languages are inconsistent. Science is different: practitioners often know that the theories they are working within, or that they are applying together to a phenomenon, are inconsistent. So here are two questions: first, how can inconsistent scientific theories be utilized in classicallogic settings? Second, what attitudes do (or should) practitioners take towards the inconsistent theories that they use?

Central to my answers to these questions is something stressed earlier in this paper: scientific reasoning doesn't occur in a formalism, but in natural languages supplemented with specialized vocabulary-specifically, in mathematized frameworks (which themselves occur in natural languages supplemented with specialized vocabulary). Formal tools_-i.e., rules of logic_-are so far off the scientific stage that they shouldn't even be seen as implicitly used by practitioners. ${ }^{24}$ Instead, scientific practitioners learn to extract valued implications from scientific theories piecemeal and laboriously: by discovering techniques and recipes of inference that can only be applied to scientific theories in specialized and highly restricted ways. ${ }^{25}$ These techniques and recipes are often — but not always — content-rich: they involve recognized idealizations of various sorts. Sometimes, however, they are computational "rules of thumb"-sheer calculational devices for manipulating a mathematical formalism that cannot be mathematically justified (at a particular time).

For our purposes, the point is this: scientists don't have a small toolkit of topicneutral logical tools (modus ponens, disjunctive syllogism, ...) that they apply to scientific theories to extract implications. That's not how scientific inference works. Rather, they have a large, and ever-growing body of topic-specific inference tools that can be only be applied to theories restricted to such and such narrow forms (or under such and such highly specialized conditions). That is, all such tools are local; they are very specific in how they can be applied to a theory, and what they will yield as a result. But the corollary that a contradictory theory can be used to derive valuable true results - without leading to an explosion of trivia-follows almost immediately.

For even if one has ways of deriving contradictory results from a theory, it won't follow that one can't apply very different ways of deriving implications from that very same theory—ones that are useful and won't yield contradictions. Indeed, exactly this is routine in empirical science. ${ }^{26}$

\footnotetext{
${ }^{24}$ I argue in my 2009 (a) that this is the case for mathematical practice: all the more so for scientific reasoning.

25 In physical theories, the problem is that the mathematics used is implicationally intractable. Partial differential equations are an excellent example of the kind of intractability I'm speaking of. Notice that the intractability of scientific laws when so represented follows rather directly from the general mathematical intractability of integration: that one can't easily manipulate integrals to extract their various implications. It's no exaggeration to say that the intractability of physical theories is due primarily to various forms of integration that are utilized. See Azzouni (2000, Part I) for discussion. It should be added, however, that most of the sentences in natural languages are, in any case, implicationally intractable as well. One has only started to grasp the magnitude of the problem when one realizes that the sentences of ordinary language-unlike first-order sentences—-"don't wear their semantics on their syntactic sleeves."

${ }^{26}$ It's common to describe scientific practitioners as "ignoring" the contradictions in their theories. Norton (2002, p. 193) asks, "what justifies ignoring all but one preferred member of a set of conclusions derived validly from postulates?" But this distorts the epistemic situation: the contradictions in such a theory may be
} 
The use I'm suggesting scientists put a contradictory scientific theory to is militantly ad hoc. Use inferential tools on it that yield results that are valuable, and avoid using inferential tools on it that yield false or contradictory results. The view also takes users of such theories to have a purely instrumental view of known contradictory theories: the theories themselves are false (because they're contradictory), although of course, the truths extracted from them are seen-obviously-as true. Compare this instrumental view with other competing views of contradictory scientific theories found in the literature. Norton (2002, pp. 193-194), for example, offers a "content driven" approach. Practitioners are taken as treating a contradictory theory as either a close approximation to a "corrected consistent theory," (a successor theory) or as an inconsistent theory from which some conclusions (or good approximations of them) of the successor theory can be extracted. I don't doubt that some uses of contradictory theories fit this pattern. ${ }^{27}$ But it's a subtle question about previous and contemporary uses of contradictory theories whether all do. ${ }^{28}$

Why should we think that scientific practice would tolerate a purely instrumental view of contradictory theories? The reason is related to why it tolerates the employment of false theories. Contrary to a long philosophical tradition, logical implications of scientific statements and theories are not epistemically transparent: they are very hard to discover. ${ }^{29}$ If, therefore, a contradictory theory lends itself successfully to such a process, scientists (who are often desperate for a way into an intractable situation) will go for it. Given enough epistemic desperation, they will do so regardless of whether the contradictory theory has any hope of approximating a successor theory, and regardless of whether the reasoning applied to a contradictory theory to yield these results has any hope of being transplanted to a consistent successor theory. ${ }^{30}$

The bottom line is that the instrumental value of a contradictory theory-like any false theory-is judged by its empirical fruits. ${ }^{31}$ This means that such a theory can be valuable even if there is no hope that it approximates - in any respectable sense-

\section{Footnote 26 continued}

revealed only when quite specific inferential techniques are applied to that theory to extract a contradiction. Other inferential tools, meanwhile, when applied to that same theory, never expose it.

27 See, e.g., Smith (1988) on Bohr's old quantum theory.

28 Norton (2002, p. 185) notes that "there is no shortage of general philosophical schemes which tolerate logical inconsistency without anarchy. What is in short supply are good case studies that can reveal clearly which of these schemes matches the actual practice of science."

29 Slogan: Reason is not epistemically transparent. The historical reasons for the contrary view are complex. There is the inheritance of an epistemic view of logic exemplified by the view that logical truths are trivial. But there is also the widespread tendency, in the twentieth century and subsequently, to treat reasoning as if it always occurs in formalisms, and indeed, in first-order formalisms.

30 What's striking about cases where a rigorous mathematical foundation is supplied for earlier inconsistent mathematical-physical inference practices (e.g., the infinitesimal calculus, the Dirac $\delta$-function) is that (usually) the earlier proof- and inference-practices don't survive. It's only the results that are-more or less-preserved. This happens even when proof- and inference-practices with earlier concepts continue to be used by practitioners "informally," a pattern that is illustrated by the use of infinitesimal and $\delta$-function reasoning in contemporary physics.

31 Laymon (1988, p. 262), describing early analyses of the Michelson-Morley experiment that utilized inconsistent assumptions, writes that "scientists were primarily interested in the computational consequences of those assumptions regardless of the consistency of those assumptions." 
a corrected noncontradictory theory. It's more or less a truism, of course, that the desired implications of a contradictory theory, being true, will transplant successfully to some corrected noncontradictory theory or other. But this is an empty truism about future scientific theories; any successor theory-if relevant-is expected to preserve empirically ratified results.

There are a number of interesting attempts to codify the "logic" of scientific reasoning within contradictory theories. ${ }^{32}$ One can either impose a version of paraconsistency or some other formalist approach that excludes triviality (e.g., reasoning from consistent subsets of an inconsistent theory, or by the adoption of "inconsistency-adaptive logics"). An instrumental interpretation of contradictory scientific theories, however, has two advantages over any attempt to so codify the reasoning in contradictory settings. There is, first, the hermeneutical point that the appropriate level of analysis of reasoning in contradictory settings must take place on the "surface" of the language. That is, the threat of triviality is deflected by the difficulty scientists have in extracting any implications from scientific theories. Second, it's clear that professionals who reason in contradictory settings recognize those settings to be pathological: they often describe their reasoning (and the reasoning of others in such settings) as sheerly computational or as ad hoc, etc. On my view, not only are practitioners right about this; we can also see why, nevertheless, the practice works.

This is related to an issue that's come up in the literature, and it's the second question I mentioned at the beginning of this section: what attitudes do (and should) practitioners take towards theories they recognize as inconsistent? Many philosophers suggest that in using an inconsistent theory, scientists must adopt some form of beliefcommitment towards it, and to this end, artificial doxastic attitudes such as "as if true," "partial" or "non-adjunctive" beliefs or belief-acquisition processes are postulated. The instrumental view I'm urging allows a much more natural attitude towards inconsistent theories, one that's exactly the same as that for false theories. These theories aren't true, one isn't committed to their truth, and one doesn't believe them. This purely instrumental attitude towards an entire inconsistent theory is compatible, as I've stressed, with positive beliefs towards (some but not all of the) implications of such theories. First, recognizing an inconsistent theory to be instrumentally valuable but false allows one to nevertheless believe true the valuable implications one can extract from that theory. ${ }^{33}$ Second, one can also-for other epistemic reasonsbelieve some of the statements of the inconsistent theory itself to be true. A sloppy way of speaking — on the part of practitioners - that can indicate this mix of beliefs is to say that the (whole) theory is "approximately true." Some philosophers have tried to give content to this idea ${ }^{34}$; but I don't see why it's needed.

When an inconsistent theory is fundamental or important enough, however, scientists are tempted to try to extract further representational content from it despite its inconsistency, and apart from its useful true implications. They are tempted, that is,

\footnotetext{
32 See, e.g., the articles in 2002 b, and especially the references therein.

33 Among these implications can be existential claims that one reads as ontologically committing, numerical approximations that one takes to be true, as well as higher-level generalizations of various sorts.

34 Among them, Da Costa and French (2002). Also see Brown (1990) for objections to the kind of instrumentalist attitude towards whole inconsistent theories that I endorse.
} 
to read off from such a theory a metaphysical picture of its subject matter. Strictly speaking, an inconsistent theory tells us nothing representational. But there are ways around this: One can (1) try to predict the overall form of a consistent successor theory, and give a representation in terms of this, one can (2) favor a consistent subset of the inconsistent theory, and give a representation from that alone, or one can (3) embrace one or another doctrine of the inconsistency of reality itself. Of course, especially in cases where the theory is clearly valued for its computational yield, one can (4) recognize ad hoc reasoning for what it is, and exclaim that no one understands what's going on, although it works. ${ }^{35}$

I have endorsed a sharp dichotomy: either belief in the truth of a (whole) theory, or an instrumental attitude towards that theory-one compatible with belief in the truth of some of its implications. I've denied the need for a belief in the "partial truth" of a theory, except as metaphor for disbelief in a whole theory coupled with belief in some of its implications. Central to my view is a principle I share with Smith (1988), one that da Costa and French describe as "unacceptable(!)" (2002, 116, their punctuation): Acceptance of the truth of a set of statements warrants their unrestricted use in all reasoning processes.

Their objection is this: "no scientific theory, past or present, receives such warrant; there are always hedging conditions, acknowledgments of limitations, approximations, etc." My response is this: if this $i$ s true, and if in all cases, these hedging conditions, acknowledgments of limitations, uses of approximations, etc., are not recognized as epistemic limitations to facilitate computational success, or as idealized reformulations of the theory so that it's easier to empirically apply, then no scientific theory, past or present, has ever been regarded as true. It's an historical question whether da Costa and French are right about this. I think they're not. ${ }^{36}$

Acknowledgments My thanks to George Smith and to an anonymous referee for valuable suggestions on an earlier version of this paper.

Open Access This article is distributed under the terms of the Creative Commons Attribution License which permits any use, distribution, and reproduction in any medium, provided the original author(s) and the source are credited.

\section{References}

Azzouni, J. (1994). Metaphysical myths, mathematical practice: The epistemology and ontology of the exact sciences. Cambridge: Cambridge University Press.

Azzouni, J. (2000). Knowledge and reference in empirical science. London: Routledge.

Azzouni, J. (2003). The strengthened liar, the expressive strength of natural languages, and regimentation. The Philosophical Forum, 34(3\&4), 329-350.

\footnotetext{
35 This was an attitude often taken by Richard Feynman.

36 Da Costa and French also complain that this principle begs the question against paraconsistent logics. It doesn't in the context of this paper: First, it appears that ordinary scientific reasoning is always compatible with codification in classical-logic terms, and second, it appears that contradictory theories are avoided when possible. Furthermore, ordinary practice universally recognizes reasoning within inconsistent theories to be ad hoc. This is enough, in my view, to rule paraconsistent approaches out of court as descriptions of ordinary scientific practice.
} 
Azzouni, J. (2004). Deflating existential commitment: A case for nominalism. Oxford: Oxford University Press.

Azzouni, J. (2006). Tracking reason: Proof, consequence, and truth. Oxford: Oxford University Press.

Azzouni, J. (2007). The inconsistency of natural languages: how we live with it. Inquiry, 50(6), 590-605.

Azzouni, J. (2009a). Why do informal proofs conform to formal norms? Foundations of Science, 14, 9-26.

Azzouni, J. (2009b). Evading truth commitments: The problem reanalyzed. Logique \& Analyse, 205, 390420 .

Azzouni, J. (2010). Talking about nothing: Numbers, hallucinations, and fictions. Oxford: Oxford University Press.

Azzouni, J. forthcoming (a). Deflationist truth. In M. Glanzberg (ed.). Handbook on truth. Oxford: Oxford University Press.

Beth, E. (1948). Naturphilosophie. Gorinchem: Noorduyn.

Brown, B. (1990). How to be realistic about inconsistency in science. Studies in History and Philosophy of Science, 21, 281-294.

Brown, B. (1992). Old quantum theory: A paraconsistent approach. Philosophy of Science Association, 2, 397-411.

Cartwright, N. (1983). How the laws of physics lie. Oxford: Oxford University Press.

Da Costa, N. C. A., \& Steven F. (2002). Inconsistency in science: A partial perspective. In J. Meheus (ed.). Inconsistency in science (pp. 105-118). New York: Kluwer Academic/Plenum Publishers.

Da Costa, N. C. A. (2003). Science and partial truth. Oxford: Oxford University Press.

Friedman, M. (1982). Review of "the scientific image". Journal of Philosophy, 74, 274-283.

Frisch, M. (2005). Inconsistency, asymmetry, and non-locality: A philosophical investigation of classical electrodynamics. Oxford: Oxford University Press.

Giere, R. (1988). Explaining science. Chicago: University of Chicago Press.

Giere, R. (2000). Theories. In W. Newton-Smith (Ed.), A companion to the philosophy of science (pp. 515-524). Oxford: Blackwell.

Kuhn, T. S. (1970). The structure of scientific revolutions. Chicago: University of Chicago Press.

Laymon, R. (1988). The Michelson-Morley experiment and the appraisal of theories. In A. Donovan (Ed.), Scrutinizing science (pp. 245-266). Dordrecht: Kluwer.

Meheus, J. (2002a). In J. Meheus (ed). How to reason sensibly yet naturally from inconsistencies (pp. 151-164). New York: Kluwer Academic Publishers.

Meheus, J. (ed.). (2002b). Inconsistency in science. Dordrecht: Kluwer Academic Publishers.

Myhill, J. (1951). On the ontological significance of the Löwenheim-Skolem theorem. In I. Copi \& J. Gould (Eds.), Contemporary readings in logical theory (1967) (pp. 40-54). New York: Macmillan.

Nickles, T. (2002). In J. Meheus (Eds.). From Copernicus to Ptolemy: Inconsistency and method. (pp. 31-33). Dordrecht: Kluwer Academic Publishers.

Norton, J. D. (2002). In J. Meheus (Eds.).A paradox in Newtonian gravitation theory II. (pp. 185-195). Dordrecht: Kluwer Academic Publishers.

Pearce, D., \& Rantala, V. (1982). Realism and formal semantics. Synthese, 52, 39-53.

Smith, J. M. (1988). Inconsistency and scientific reasoning. Studies in History and Philosophy of Science, $19,429-445$.

Sneed, J. (1971). The logical structure of mathematical physics. Dordrecht: Reidel.

Stegmüller, Wolfgang. (1976). The structure and dynamics of theories. New York: Springer.

Stegmüller, W. (1978). A combined approach to the dynamics of theories. Theory and Decision, 9, 39-75.

Suppe, F. (1977). The structure of scientific theories. Urbana: University of Illinois Press.

Suppe, F. (1989). The semantic conception of theories and scientific realism. Chicago: University of Illinois Press.

Suppes, P. (1967). What is a scientific theory? In S. Morgenbesser (Ed.), Philosophy of science today (pp. 55-67). New York: Basic Books.

Tarski, A. (1983). The concept of truth in formalized languages. In J. Corcoran (Ed.). Logic, semantics, metamathematics, $2^{\text {nd }}$ edition (pp. 152-278). Indianapolis, IN: Hackett Publishing Company Inc.

Van Fraassen, B. (1980). The scientific image. Oxford: Oxford University Press.

Van Fraassen, B. (1985). Empiricism in the philosophy of science. In P. Churchland \& C. Hooker (Eds.), Images of science (pp. 245-308). Chicago: University of Chicago Press.

Van Fraassen, B. (1989). Laws and symmetry. Oxford: Oxford University Press.

Worrell, J. (1984). 'An unreal image', Review of van Fraassen, The scientific image. British Journal for the Philosophy of Science, 35, 65-80. 